OAI-PMH: http://www.indteca.com/ojs/index.php/Revista Scientific/oai

Artículo Original / Original Article

\title{
Una mirada desde el Síndrome de Burnout hacia la Gerencia Integral en el Siglo XXI
}

\author{
Autor: Carlos Liborio Camacho Quintero \\ Universidad de Los Andes, ULA \\ ccamacho@ula.ve; clcamachoq71@gmail.com \\ Mérida, Venezuela \\ https://orcid.org/0000-0002-7552-5245
}

Resumen

El presente artículo tiene como objetivo teorizar el síndrome de burnout en la gerencia integral del siglo XXI presentado por el personal administrativo del Instituto de Geografía de la Universidad de Los Andes como respuesta prolongada del estrés laboral ante los factores de cansancio o agotamiento emocional; con la intención de optimizar el desempeño de los trabajadores de dicha institución, mediante el diagnóstico se observó que existe conflictos interpersonales que contribuyen al deterioro organizacional, razón por la cual la propuesta de una estrategia gerencial integral para la prevención de dicho síndrome se enfoca en canalizar las emociones. Metodológicamente se enmarco en un paradigma cualitativo, apoyado en una investigación de tipo etnometodológica como una solución posible a un problema de tipo práctico. Se pretende hacer una investigación para medir el grado de desgaste profesional u ocupacional de cada empleado, para evaluar así, si el personal posee estrés laboral excesivo negativo el cual puede conllevar a un distrés debido a la acumulación de situaciones estresantes. Se recomendó disminuir utilizar mediante tácticas para tratar las inconformidades de los trabajadores y a su vez mejorar el clima laboral mediante la reactivación de las actitudes productivas del personal, la conformación de equipos de trabajo, motivación e incentivos y delegación de tareas. Posteriormente, se exhorta que esta propuesta pueda aplicarse y difundirse en otras organizaciones preocupadas por el desarrollo y el desempeño de su personal y así garantizar su crecimiento laboral.

Palabras clave: efectos fisiológicos; enfermedad profesional; estrés mental; actitud laboral.

Fecha de Recepción: 22-12-2018
Fecha de Aceptación: 05-06-2019
Fecha de Publicación: 05-08-2019 


\title{
A look from the Burnout Syndrome to the Integral Management in the XXI Century
}

\begin{abstract}
The objective of this article is to theorize the burnout syndrome in the integral management of the 21 st century presented by the administrative staff of the Institute of Geography of the University of Los Andes as a prolonged response to work stress in the face of fatigue or emotional exhaustion; with the intention of optimizing the performance of the workers of said institution, through the diagnosis it was observed that there are interpersonal conflicts that contribute to organizational deterioration, reason why the proposal of a comprehensive management strategy for the prevention of said syndrome focuses on channeling the emotions. Methodologically, it is framed in a qualitative paradigm, supported by ethnomethodological research as a possible solution to a practical problem. It is intended to do an investigation to measure the degree of professional or occupational wear of each employee, to evaluate this way, if the personnel has excessive negative work stress which can lead to a distress due to the accumulation of stressful situations. It was recommended to decrease using tactics to address the nonconformities of workers and in turn improve the working environment by reactivating the productive attitudes of the staff, the formation of work teams, motivation and incentives and delegation of tasks. Subsequently, it is exhorted that this proposal can be applied and disseminated in other organizations concerned about the development and performance of their staff and thus ensure their job growth.
\end{abstract} work attitude.

Keywords: physiological effects; occupational diseases; mental stress;

Date Received: 22-12-2018
Date Acceptance:

05-06-2019
Date Publication:

05-08-2019 


\section{Introducción}

En la actualidad las teorías vinculadas al estrés han cobrado auge en la gerencia integral del siglo XXI, la cual es estimulada por contextos angustiosos que producen perturbaciones psicológicas y psicosomáticas que son nocivas al ser humano, asimismo una persona estresada confronta escenarios externos amenazantes o desafiantes, las cuales resultan difíciles de sobreponer, asimismo perturban el equilibrio emocional entorpeciendo su desempeño ya sea personal o laboral. De la misma perspectiva, Schwartzmann (2004), señala:

El estrés es un efecto que se origina al reaccionar ante ciertos eventos, donde el cortisol que es la hormona del estrés actúa en el cuerpo produciendo un entorno arduo con encauzamiento, fuerza y astucia, todo este se conoce como respuesta al estrés (págs. 174-184).

Por tal esbozo, el eustrés o estrés positivo es necesario porque es un indicativo que motiva al ser humano al logro sin exceder en actividades ya que puede ser perjudicial y causar problemas cuando se exagera, lo perjudicial es de gran preocupación afectando el rendimiento y el nivel de producción tanto en los empleados como en la organización. En este sentido, Ivancevich, Konopaske y Matteson (2006): consideran el estrés profesional como una "respuesta adaptativa moderada por las diferencias individuales, consecuencia de cualquier acción, situación o suceso, que impone exigencias especiales a una persona" (pág. 58).

Si el clima organizacional es tanto placentero como seductor se alcanza el bienestar del talento humano, esta sinergia es una simbiosis para el logro de efectividad y productividad. Cabe enfatizar, que la higiene y seguridad laboral es necesaria integrarla al sitio laboral porque la salud física mejora las condiciones externas del organismo humano, cuando esto ocurre el clima organizacional se transforma en saludable optimizando positivamente el 
bienestar del talento humano, estimulando mejores condiciones que impulse la salud mental, psicológica y sociales que actúen como medicina efectiva soslayando riesgos emocionales como es el caso del estrés laboral.

En el área administrativa del Instituto de Geografía los empleados muestran una diligencia dinámica, aun cuando se enfrentan a exigencias y restricciones del contexto que prevalecen para mantenerlas bajo control; por ello, el ambiente laboral no es el adecuado, sobre todo por la presión en su actividad profesional, lo que genera reacción del estrés, esto motivado al salario emocional que produce ausentismo y desmotivación laboral.

En base a la indagación recolectada y suministrada en el proceso de exploración, se atisba una predisposición interna en el Instituto mencionado, una de las principales debilidades que presentan estos empleados son las actividades coercitivas rigiéndose por pautas preestablecidas, es decir, ellos deben cumplir con el volumen de trabajo, ajustándose al tiempo estipulado por la administración central, además, adaptarse al desempeño y rendimiento que se les exige en el cumplimiento de sus funciones, el cual en estos momentos es complicado por la situación económica que vive Venezuela entre los años 2014 y 2018, ocasionando fatigas irrecuperables, desinterés por el trabajo y enfermedades ocupacionales, como es el estrés laboral que lesiona la vida y salud de los empleados, conllevando al indiscutible punto de esta investigación, el distrés que es el estrés dañino generando ergofobia y por ende el síndrome de burnout.

Esta situación minimiza la productividad del trabajo, por lo tanto, es necesario mantener un control efectivo mediante el diseño de estrategias gerenciales que se efectúen para reducir el impacto y margen de los factores estresantes que están sometidos el personal administrativo del instituto en estudio, sin embargo, es necesario analizar las causas que originan el síndrome en estos empleados; generándose las siguientes interrogantes: ¿Cuáles son las actividades que realiza el personal administrativo que labora 
en el departamento administrativo del Instituto de Geografía de la Universidad de Los Andes?; ¿Cuáles son los factores estresantes asociados al trabajo que realiza el personal administrativo?; ¿Qué estrategias se pueden implementar para evitar el estrés laboral del personal administrativo?; ¿Se puede evitar el quemado de un profesional en cualquier área organizacional del Instituto de Geografía de la Universidad de Los Andes?

Como objetivo general, se teoriza el síndrome de burnout hacia la gerencia integral en el siglo XXI, presentado por el personal administrativo del Instituto de Geografía de la Universidad de Los Andes, para construir lineamientos estratégicos gerenciales, mediante tácticas para combatir las inconformidades de los empleados, enfocada en el uso de la canalización de las emociones; para así, poder reorientar la gerencia administrativa universitaria, con el fin de impulsar el desarrollo organizacional e integración del capital humano para un mejor desempeño en un ambiente que contribuya a minimizar el estrés que afecta la salud.

\section{Referentes Teóricos}

Entrando en materia, las investigadoras Suárez y Contreras (2010), presentan un trabajo intitulado: "Síndrome de burnout: comparación entre alumnos y profesor odontólogo en la Facultad de Odontología de la Universidad de Los Andes" (pág. 12). El propósito de la investigación se centra en las características sociológicas para examinar si el síndrome está presente generando estrés crónico, porque es un problema de salud pública que está afectando cada vez más al estudiante como al profesor, los resultados demostraron que en el personal de la facultad de odontología presentan fatiga generando graves lesiones de salud, igualmente utilizaron la metodología cuantitativa de tipo descriptivo y documental de campo, con una población de 50 estudiantes, donde tomaron como muestra a 5 odontólogos 
espontáneamente, de la misma manera el método manejado es el cuestionario de Maslach, utilizado para profesionales y estudiantes de la Facultad de Odontología de la Universidad de Los Andes, posteriormente los hallazgos se analizaron mediante la estadística descriptiva utilizando el programa estadístico informático SPSS versión 18.

\subsection{Contexto Teórico}

En relación con las bases teóricas, cabe destacar que el presente artículo científico se dimensiono a partir del análisis de los fundamentos doctrinarios siguientes: Estrés, consecuencias desde la perspectiva laboral, enfermedad que se traduce en distrés, eustrés, síndrome del quemado o burnout, ergofobia, evolución, manifestaciones, entre otras.

Según Stora (1991): "los agentes estresantes pueden originarse en el interior de la persona o en su ambiente externo. Estos últimos pueden provenir del mundo profesional o extra profesional" (pág. 10); es decir, puede causar síntomas físicos cuando se prolonga por mucho tiempo, así como una respuesta a los retos de la vida diaria y los cambios. En ese sentido, para los especialistas, el detonante común de este tipo de ansiedad incontrolable son las exigencias que sobrepasan los recursos de cada persona, o cuando es inducido por un agente estresor.

Existen estudios que plantean que trabajos psicológicamente muy demandantes, con escaso control del trabajador sobre el proceso productivo, se asocian a riesgo aumentando las enfermedades cardiovasculares; específicamente se menciona hipertensión arterial, infarto al miocardio y accidente vascular cerebral. Con respecto a las Enfermedades Músculo esqueléticas: existe una opinión ampliamente extendida en especialistas de salud ocupacional acerca de que el estrés laboral aumenta el riesgo de sufrir lumbago y lesión de extremidades superiores (tendinitis), igualmente Gil (2005a), mencionan que el burnout se transforma en síndrome del carbonizado 
esbozando:

Como ellos denominan a los individuos que padecen este problema. De ahí, que en la actualidad una traducción libre de burnout sea la de quemado, cuando realmente lo que se está reflejando es una situación cualitativamente más grave llegando a carbonizarse (págs. 36-37).

Estos mismos autores plantean que burnout tal vez pueda ser descrito como el estado mental y físico resultante de los efectos de debilitamiento experimentados por sensaciones negativas prolongadas, relacionadas con el trabajo y el valor que le merece al empleado el "cara a cara" del trabajo y de los compañeros, asimismo, proponen cuatro fases por las cuales pasa todo individuo con burnout: 1. Entusiasmo, caracterizado por elevadas aspiraciones, energía desbordante y carencia de peligro; 2. Estancamiento, que surge tras no cumplirse las expectativas originales, empezando a aparecer la frustración; 3. Frustración, en la que comienzan a surgir problemas emocionales, físicos y conductuales. Esta fase sería el núcleo central del síndrome; y 4. Apatía, que sufre el individuo y que constituye el mecanismo de defensa ante la frustración.

En esta misma época, Gillespie (1980), citado por Gil (2005b), intentando resolver la ambigüedad definicional, que según el autor existe, clasifica al burnout según dos tipos claramente diferenciados: "burnout activo, que se caracterizaría por el mantenimiento de una conducta asertiva, y burnout pasivo en el que predominarían los sentimientos de retirada y apatía" (pág. 21). El activo tendría que ver, fundamentalmente, con factores organizacionales o elementos externos a la profesión, mientras que el pasivo se relacionaría con factores internos psicosociales. El autor abre, de esta forma, la posibilidad de la existencia de varias manifestaciones del burnout que, posteriormente, otros autores retomarían para intentar explicar la complejidad del síndrome. 


\section{Contexto Metodológico}

\subsection{Materiales y Métodos}

Principalmente, la investigación se canalizo en un modelo cualitativo de tipo etnometodológico, donde Heritage (1998), citada por Gil (2007): lo define como el "estudio del cuerpo del conocimiento de sentido común y de la gama de procedimientos y consideraciones por medio de las cuales los miembros corrientes de la sociedad dan sentido a las circunstancias y actúan en consecuencia" (pág. 25); por ello es necesario diseñar una estrategia gerencial para minimizar la presencia del síndrome del burnout, asimismo el personal administrativo del Instituto de Geografía de la Universidad de Los Andes busca canalizar las emociones, como técnicas y procedimientos se utilizó el cuestionario de Maslach.

\subsection{Informantes Clave}

Los informantes clave objeto de estudio de la presente investigación es el personal administrativo adscrito al Instituto de Geografía de la Universidad de Los Andes (ULA), Mérida, Núcleo Forestal, estando constituida por cinco (5) empleados de oficina que laboran en las instancias administrativas del área de estudio, a saber, la Dirección, la Secretaría y la oficina administrativa, estos últimos ubicados en un área adyacente a la Dirección.

\subsection{Recolección de Datos}

Al respecto, Arias (2012): "se entiende por las técnicas de investigación, el procedimiento o forma particular de obtener datos o información" (pág. 67). En este sentido el investigador consigue mediante la aplicación de las técnicas la adquisición de la recolección de la data, acopiando información pertinente sobre las variables y contextos involucrados en la investigación, asimismo, es indispensable utilizar instrumentos como la observación directa y el cuestionario Maslach, de forma auto aplicada y autodidactica para medir así el 
desgaste profesional de cada empleado, un tiempo estipulado entre 10 y 15 minutos, la escala es consistente y de alta fiabilidad con una puntuación decimal de 0,9 y está constituida por 22 ítems para este caso.

Por lo tanto, el cuestionario Maslach está constituido por 22 ítems en forma de aseveraciones, enfocándose en los sentimientos y actitudes del personal administrativo y medir si existe desgaste, cuya finalidad es observar la frecuencia e intensidad con la que se sufre del síndrome de burnout. Para Miravalles (2009): "el uso de la técnica de Maslach permitió conseguir la información necesaria, de manera simple y directa, respondidas por las personas objeto de estudio" (pág. 21). El instrumento de recolección de datos usado fue dicho cuestionario, destinado al personal administrativo del Instituto de Geografía de la Universidad de Los Andes.

\section{Resultados}

El proceso de investigación se da a conocer de forma organizada los pasos desarrollados para obtener la información de las tablas y cuadros generados a través del cuestionario de Maslach, de igual modo los informantes clave se contrasta para medir los 3 aspectos del síndrome de burnout comenzando por el cansancio emocional de vital importancia, continuando con la despersonalización y la realización personal, tomando como referencia los objetivos de la investigación. El cuestionario, según Hurtado y Toro (2010), señalan que: "...es el formulario que contiene las preguntas o variables de la investigación y en el que se registran las respuestas de los encuestados..." (pág. 74). El cuestionario fue aplicado a cada trabajador administrativo del Instituto de Geografía de la Universidad de Los Andes (ULA), observándose en la Tabla 1, los informantes clave. 
Tabla 1. Personal del Instituto de Geografía (ULA)

\begin{tabular}{|l|l|}
\hline Director & 1 \\
\hline Secretaria & 1 \\
\hline Administradora & 1 \\
\hline Asistente Contable & 1 \\
\hline Asistente Administrativa & 1 \\
\hline
\end{tabular}

Fuente: El Autor (2019).

\subsection{Cuestionario Maslach efectuado al personal administrativo del Instituto de Geografía de la Universidad de Los Andes.}

El cuestionario Maslach tiene un tiempo de duración que oscila entre 10 a 15 minutos, consiste en medir 3 aristas del síndrome como lo es el cansancio emocional, después viene la despersonalización y por último la realización personal, es necesario puntualizar cada arista para verificar en que grado está presente este síndrome y así diagnosticar el trastorno, se visualiza el Cuestionario en la Tabla 2.

Tabla 2. Cuestionario de MASLACH.

\begin{tabular}{|c|l|}
\hline 1 & $\begin{array}{l}\text { Emocionalmente me siento agotado por mi trabajo en el Instituto de Geografía de la } \\
\text { ULA }\end{array}$ \\
\hline 2 & $\begin{array}{l}\text { Me siento cansado al final de la jornada de trabajo en el Instituto de Geografía de la } \\
\text { ULA. }\end{array}$ \\
\hline 3 & $\begin{array}{l}\text { Cuando me levanto por la mañana y me enfrento a otra jornada de trabajo me siento } \\
\text { fatigado. }\end{array}$ \\
\hline 4 & $\begin{array}{l}\text { Tengo facilidad para comprender como se sienten mis compañeros del Instituto de } \\
\text { Geografía de la ULA. }\end{array}$ \\
\hline 5 & $\begin{array}{l}\text { Estoy tratando a algunos compañeros del Instituto de Geografía de la ULA como si } \\
\text { fueran objetos impersonales. }\end{array}$ \\
\hline 6 & $\begin{array}{l}\text { Siento que trabajar todo el día con mis compañeros supone un gran esfuerzo y me } \\
\text { cansa. }\end{array}$ \\
\hline 7 & $\begin{array}{l}\text { Trato con mucha eficacia los problemas de mis compañeros en el Instituto de } \\
\text { Geografía de la ULA. }\end{array}$ \\
\hline 8 & Siento que mi trabajo me está desgastando y me estoy quemando por mi trabajo. \\
\hline 9 & $\begin{array}{l}\text { Creo que con mi trabajo estoy influyendo positivamente en la vida de mis compañeros } \\
\text { en el Instituto de Geografía de la ULA }\end{array}$ \\
\hline 10 & Me he vuelto más insensible con la gente desde que ejerzo mi profesión. \\
\hline 11 & Pienso que este trabajo me está endureciendo emocionalmente. \\
\hline 12 & Me siento con mucha energía en mi trabajo. \\
\hline
\end{tabular}




\begin{tabular}{|c|l|}
\hline 13 & Me siento frustrado en mi trabajo en el Instituto de Geografía de la ULA. \\
\hline 14 & Creo que trabajo demasiado en el Instituto de Geografía de la ULA. \\
\hline 15 & $\begin{array}{l}\text { No me preocupa realmente lo que les ocurra a algunos de mis compañeros en el } \\
\text { Instituto de Geografía de la ULA. }\end{array}$ \\
\hline 16 & $\begin{array}{l}\text { Trabajar directamente con mis compañeros me produce estrés en el Instituto de } \\
\text { Geografía de la ULA. }\end{array}$ \\
\hline 17 & $\begin{array}{l}\text { Siento que puedo crear con facilidad un clima agradable con mis compañeros en el } \\
\text { Instituto de Geografía de la ULA. }\end{array}$ \\
\hline 18 & $\begin{array}{l}\text { Me siento motivado después de trabajar en contacto con mis compañeros en el } \\
\text { Instituto de Geografía de la ULA. }\end{array}$ \\
\hline 19 & $\begin{array}{l}\text { Creo que consigo muchas cosas valiosas en este trabajo en el Instituto de Geografía } \\
\text { de la ULA. }\end{array}$ \\
\hline 20 & Me siento acabado en mi trabajo, al límite de mis posibilidades. \\
\hline 21 & $\begin{array}{l}\text { En mi trabajo en el Instituto de Geografía de la ULA trato los problemas } \\
\text { emocionalmente con mucha calma. }\end{array}$ \\
\hline 22 & $\begin{array}{l}\text { Creo que mis compañeros me culpan de algunos de sus problemas en el Instituto de } \\
\text { Geografía de la ULA }\end{array}$ \\
\hline
\end{tabular}

Fuente: El Autor (2019).

Cansancio emocional: El cansancio emocional o agotamiento del personal administrativo del Instituto de Geografía de la Universidad de Los Andes, se asocia con sentimientos de angustia, estrés y depresión, igualmente se analiza los problemas de atención y sensación laboral de los valores del personal Administrativo del Instituto de Geografía de la ULA. Que se observan en la Tabla 3.

Tabla 3. Cansancio Emocional.

1.- Emocionalmente me siento agotado por mi trabajo en el Instituto de Geografía de la ULA 2.- Me siento cansado al final de la jornada de trabajo en el Instituto de Geografía de la ULA.

3.- Cuando me levanto por la mañana y me enfrento a otra jornada de trabajo me siento fatigado.

6.- Siento que trabajar todo el día con mis compañeros/as supone un gran esfuerzo y me cansa.

8.- Siento que mi trabajo me está desgastando y me estoy quemando por mi trabajo. 13.- Me siento frustrado en mi trabajo en el Instituto de Geografía de la ULA.

14.- Creo que trabajo demasiado en el Instituto de Geografía de la ULA

16.- Trabajar directamente con mis compañeros me produce estrés en el Instituto de Geografía de la ULA. 
20.- Me siento consumado en mi trabajo al límite de mis posibilidades.

\begin{tabular}{|c|c|c|c|c|}
\hline Director & 14 & \multirow{5}{*}{$\begin{array}{c}\text { Bajo 0-18 } \\
\text { Medio 19-26 } \\
\text { Alto } 27-54\end{array}$} & Baja & \multirow{5}{*}{$\begin{array}{c}\text { Nivel de cansancio } \\
\text { emocional }\end{array}$} \\
\hline Secretaria & 36 & & Alta & \\
\hline Administradora & 33 & & Alta & \\
\hline Asistente Contable & 29 & & Alta & \\
\hline $\begin{array}{c}\text { Asistente } \\
\text { Administrativo }\end{array}$ & 21 & & Media & \\
\hline
\end{tabular}

Fuente: El Autor (2019).

Despersonalización: El trastorno de despersonalización es un trastorno de personalidad caracterizado por la experimentación de graves sentimientos de irrealidad que dominan la vida de la persona y que evitan el funcionamiento normal en la vida, en este cuadro se observan el nivel de despersonalización del personal administrativo del Instituto de Geografía de la Universidad de Los Andes visualizándose en la Tabla 4.

Tabla 4. Despersonalización.

\begin{tabular}{|c|c|c|c|c|}
\hline \multicolumn{5}{|c|}{$\begin{array}{l}\text { 5.- Creo que estoy tratando a algunos compañeros/as como si fueran objetos } \\
\text { impersonales. }\end{array}$} \\
\hline \multicolumn{5}{|c|}{ 10.- Me he vuelto más insensible con la gente desde que ejerzo mi profesión. } \\
\hline \multicolumn{5}{|c|}{ 11.- Pienso que este trabajo me está endureciendo emocionalmente. } \\
\hline \multicolumn{5}{|c|}{ 15.- No me preocupa realmente lo que les ocurra a algunos de mis compañeros. } \\
\hline \multicolumn{5}{|c|}{ 22.- Creo que mis compañeros me culpan de algunos de sus problemas. } \\
\hline Director & 9 & \multirow{5}{*}{$\begin{array}{c}\text { Bajo 0-18 } \\
\text { Medio 19-26 } \\
\text { Alto } 27-54\end{array}$} & Alta & \multirow{5}{*}{$\begin{array}{c}\text { Nivel de } \\
\text { Despersonalización }\end{array}$} \\
\hline Secretaria & 12 & & Alta & \\
\hline Administradora & 11 & & Alta & \\
\hline Asistente Contable & 15 & & Alta & \\
\hline Asistente Administrativo & 8 & & Alta & \\
\hline
\end{tabular}

Fuente: El Autor (2019).

Realización personal: La realización personal se consigue cuando el trabajador puede realizar con normalidad sus aspiraciones personales en condiciones óptimas laborales con sus compañeros de trabajo, buscando un equilibrio emocional, valores morales y un sentido lúdico de la vida, por lo 
tanto, en la Tabla 5, se representan los niveles de realización del personal administrativo del Instituto de Geografía de la Universidad de Los Andes.

Tabla 5. Realización personal.

\begin{tabular}{|c|c|c|c|c|}
\hline \multicolumn{5}{|c|}{ 4.- Tengo facilidad para comprender como se sienten mis compañeros. } \\
\hline \multicolumn{5}{|c|}{ 7.- Creo que trato con mucha eficacia los problemas de mis compañeros. } \\
\hline \multicolumn{5}{|c|}{ 9.- Creo que con mi trabajo estoy influyendo positivamente en la vida de mis compañeros. } \\
\hline \multicolumn{5}{|c|}{ 12.- Me siento con mucha energía en mi trabajo. } \\
\hline \multicolumn{5}{|c|}{ 17.- Siento que puedo crear con facilidad un clima agradable con mis compañeros. } \\
\hline \multicolumn{5}{|c|}{ 18.- Me siento motivado después de trabajar en contacto con mis compañeros. } \\
\hline \multicolumn{5}{|c|}{ 19.- Creo que consigo muchas cosas valiosas en este trabajo. } \\
\hline \multicolumn{5}{|c|}{ 21.- Creo que mis compañeros me culpan de algunos de sus problemas. } \\
\hline Director & 11 & Valores & Baja & \multirow{5}{*}{$\begin{array}{l}\text { Nivel de realización } \\
\text { personal }\end{array}$} \\
\hline Secretaria & 27 & \multirow{4}{*}{$\begin{array}{c}\text { Bajo 0-18 } \\
\text { Medio 19-26 } \\
\text { Alto } 27-54\end{array}$} & Alta & \\
\hline Administradora & 32 & & Alta & \\
\hline Asistente Contable & 31 & & Alta & \\
\hline Asistente Administrativo & 12 & & Media & \\
\hline
\end{tabular}

Fuente: El Autor (2019).

Se puede observar en la Tabla 6, los niveles presentados por el personal administrativo del Instituto de Geografía de la Universidad de Los Andes basado en las variables del cansancio emocional, despersonalización y realización personal exteriorizado en sus actividades diarias.

Tabla 6. Análisis de los Niveles del Síndrome de Burnout.

\begin{tabular}{|c|c|c|c|}
\hline Síndrome de Burnout & $\begin{array}{c}\text { Cansancio } \\
\text { emocional }\end{array}$ & Despersonalización & $\begin{array}{c}\text { Realización } \\
\text { personal }\end{array}$ \\
\hline Director & Baja & Alta & Baja \\
\hline Secretaria & Alta & Alta & Alta \\
\hline Administradora & Alta & Alta & Alta \\
\hline Asistente Contable & Alta & Alta & Baja \\
\hline $\begin{array}{c}\text { Asistente } \\
\text { Administrativo }\end{array}$ & Media & Alta & Alta \\
\hline
\end{tabular}

Fuente: El Autor (2019). 


\section{Propuesta}

\subsection{Estrategia gerencial integral para la canalización de las emociones}

aplicada al personal administrativo del Instituto de Geografía de la Universidad de Los Andes.

El articulo surge como motivo del aumento de las exigencias en las cuales están sometidos actualmente los profesionales que trabajan en espacios cerrados, manifestándose el síndrome de burnout en el personal administrativo del mencionado Instituto, que induce en la salud y es perjudicial en la calidad de vida.

Por esta razón, la propuesta es una simbiosis para minimizar el síndrome de burnout añadiendo variables laborales al personal administrativo que laboran en espacios cerrados, sin embargo, el objetivo fundamental es desarrollar estrategias gerenciales para minimizar el estrés, mediante tácticas para tratar las inconformidades de los trabajadores enfocada en el uso de la canalización de las emociones; ya que, la idea es determinar la frecuencia y factores de riesgo de dicho síndrome presentado por el personal administrativo del Instituto de Geografía de la Universidad de Los Andes.

\subsection{Estructura}

La presente propuesta se encuentra estructura en tres fases (03) que ayudaran el desarrollo de esta. A continuación, se describen las tres fases que la conforman:

Fase 1. Diagnóstico: Esta Fase comprende el diagnóstico y sondeo tanto de las estrategias que se deben aplicar como de las emociones que se deben canalizar, donde se diseña en la Tabla 7, un modelo operativo para incentivar y motivar al personal administrativo del Instituto de Geografía de la Universidad de Los Andes (ULA). 
Tabla 7. Diagnóstico de las estrategias para el personal Administrativo del Instituto de Geografía de la ULA

\begin{tabular}{|c|c|c|c|c|}
\hline Objetivos & Responsable & $\begin{array}{l}\text { Recursos } \\
\text { Usados }\end{array}$ & $\begin{array}{c}\text { Actividad por } \\
\text { plan } \\
\text { operativo }\end{array}$ & $\begin{array}{l}\text { Metas por } \\
\text { actividad }\end{array}$ \\
\hline $\begin{array}{l}\text { Uso de programas } \\
\text { contables para } \\
\text { maximización del } \\
\text { proceso. }\end{array}$ & \multirow{3}{*}{$\begin{array}{c}\text { Directora y } \\
\text { Administradora }\end{array}$} & $\begin{array}{l}\text { Programa } \\
\text { SUAS ULA }\end{array}$ & $\begin{array}{l}\text { Capacitar al } \\
\text { personal para } \\
\text { el uso del } \\
\text { software }\end{array}$ & $\begin{array}{l}\text { Maximizar y } \\
\text { agilizar el } \\
\text { proceso de } \\
\text { gestión }\end{array}$ \\
\hline $\begin{array}{c}\text { Recompensación } \\
\text { por sobretiempos } \\
\text { y dedicación a la } \\
\text { organización. }\end{array}$ & & $\begin{array}{c}\text { Libreta y } \\
\text { recursos } \\
\text { audiovisuales. }\end{array}$ & $\begin{array}{l}\text { Capacidades } \\
\text { y dedicación } \\
\text { mostrada dar } \\
\text { recompensas }\end{array}$ & $\begin{array}{c}\text { Los mejores } \\
\text { trabajadores } \\
\text { para motivar e } \\
\text { incentivar. }\end{array}$ \\
\hline $\begin{array}{c}\text { Establecer } \\
\text { políticas de } \\
\text { incentivo al } \\
\text { personal. }\end{array}$ & & $\begin{array}{l}\text { Reuniones, } \\
\text { discusiones. }\end{array}$ & $\begin{array}{c}\text { Reuniones y } \\
\text { discusiones } \\
\text { para estimular } \\
\text { nuevas } \\
\text { políticas. }\end{array}$ & $\begin{array}{c}\text { Políticas que } \\
\text { beneficien a los } \\
\text { trabajadores } \\
\text { para evitar } \\
\text { resentimientos } \\
\text { laborales. }\end{array}$ \\
\hline
\end{tabular}

Fuente: El Autor (2019).

Fase 2. Planeación: La planeación consta en la Tabla 8 , de una presentación esquematizada para el logro de los objetivos específicos, donde se observa como menguar el manejo creativo del estrés cuya finalidad es trabajar en equipo para consolidar las relaciones interpersonales, mejorar la comunicación y minimizar los conflictos.

Tabla 8. Diagnóstico de las estrategias para el personal Administrativo del Instituto de Geografía de la ULA

\begin{tabular}{|c|c|c|c|}
\hline Contenido & Actividades & $\begin{array}{l}\text { Recursos } \\
\text { Usados }\end{array}$ & Responsable \\
\hline $\begin{array}{l}\text { - Angustia, cansancio y estrés. } \\
\text { - Éxito y estrés. } \\
\text { - Circunstancias que precipitan } \\
\text { el estrés. } \\
\text { - Claves del manejo del estrés. } \\
\text { - Resolver la tensión }\end{array}$ & $\begin{array}{l}\text { Apertura } \\
\text { Dinámica } \\
\text { Desarrollo }\end{array}$ & $\begin{array}{l}\text { Salón de } \\
\text { reuniones } \\
\text { Papelería } \\
\text { Recursos }\end{array}$ & $\begin{array}{l}\text { Director y } \\
\text { Administradora }\end{array}$ \\
\hline
\end{tabular}




\begin{tabular}{|c|c|c|}
\hline $\begin{array}{l}\text { - Técnicas de relajación y de } \\
\text { control mental. } \\
\text { - Salario emocional positivo. } \\
\text { - Salario emocional negativo. } \\
\text { - Estrategias efectivas del } \\
\text { estrés. }\end{array}$ & $\begin{array}{l}\text { Dinámica } \\
\text { Practica } \\
\text { Conclusiones }\end{array}$ & Audiovisuales \\
\hline
\end{tabular}

Fuente: El Autor (2019).

Fase 3. Desarrollo: Elaboración de un método gerencial para la canalización de las emociones en el personal administrativo del Instituto de Geografía de la Universidad de Los Andes, donde en la Tabla 9, se utilizan estrategias basadas en sinergia laboral para crear habilidades para el manejo de emociones personales proyectadas al equipo administrativo del Instituto de Geografía de la Universidad de Los Andes (ULA).

Tabla 9. Método gerencial para el personal Administrativo del Instituto de Geografía de la ULA

\begin{tabular}{|c|c|c|c|}
\hline $\begin{array}{l}\text { Desarrollo de } \\
\text { talleres y } \\
\text { cursos }\end{array}$ & $\begin{array}{l}\text { Actividades de } \\
\text { integración }\end{array}$ & Pizarra Emocional & $\begin{array}{c}\text { Buzón de } \\
\text { sugerencias para } \\
\text { trabajadores }\end{array}$ \\
\hline $\begin{array}{l}\text { - Capacitación } \\
\text { constante al } \\
\text { personal } \\
\text { administrativo. } \\
\text { - Seguimiento a } \\
\text { través de una } \\
\text { encuesta en la } \\
\text { cual se mida la } \\
\text { influencia que } \\
\text { ésta ha logrado } \\
\text { en los } \\
\text { trabajadores. }\end{array}$ & $\begin{array}{l}\text { Actividades de } \\
\text { relajación como } \\
\text { riso terapia, bailo } \\
\text { terapia, } \\
\text { dinámicas de } \\
\text { trabajo en equipo } \\
\text { y clases de } \\
\text { CrossFit. }\end{array}$ & $\begin{array}{l}\text { Crear una cartelera con } \\
\text { los nombres de cada } \\
\text { uno de los empleados, } \\
\text { en la cual se tengan } \\
\text { diferentes caritas con } \\
\text { las expresiones de: } \\
\text { Alegría, Tristeza, } \\
\text { Agotamiento, Molestia. }\end{array}$ & $\begin{array}{l}\text { Es un medio a través } \\
\text { del cual los } \\
\text { empleados podrán } \\
\text { formular ideas, } \\
\text { inquietudes, } \\
\text { soluciones para } \\
\text { resolver algún } \\
\text { conflicto interno, una } \\
\text { propuesta que deseen } \\
\text { realizar, entre otras. }\end{array}$ \\
\hline
\end{tabular}

Fuente: El Autor (2019). 


\section{Reflexiones}

El propósito de esta investigación es conocer el nivel de burnout presentado en los empleados del Instituto de Geografía de la Universidad de Los Andes porque estos laboran en espacios cerrados y, a su vez, determinar los factores que lo ocasionan.

Sin embargo, se observa que el $70 \%$ de los trabajadores padece altos niveles de burnout. Cabe destacar, que estudios previos a este síndrome comprueban que más de la mitad de la muestra objeto de estudio padece altos niveles de este síndrome.

Asimismo, es importante destacar que los trabajadores con más de cinco años laborando en el espacio especificado, presentan niveles superiores de burnout que los más experimentados, igualmente el menor nivel de burnout es motivado a que el personal administrativo ha aprendido a controlar las emociones negativas y a promover las positivas debido a la rutina laboral para evitar bloqueos mentales.

De acuerdo con lo investigado, se deduce que, según el tipo de cargo de estos trabajadores, presentan un nivel más elevado de burnout y se encuentran más agotados emocionalmente que aquellos con cargo con menos nivel de exigencia. También, aquellos trabajadores que tienen algún tipo de descanso o pausa de las tareas organizacionales, pautado cerca de la fecha, presentan un nivel de burnout menos elevado, como muestra: vacaciones o permisos remunerados.

Esto va a posibilitar el diseño y la elaboración de planes de prevención, atención temprana y disminución del burnout, para facilitar así el bienestar de estos profesionales. Por esto, la propuesta de usar la Inteligencia Emocional como estrategia para el manejo y canalización de las emociones aplicada al personal del Instituto de Geografía de la Universidad de Los Andes. 


\section{Recomendaciones}

1. Capacitar al personal, motivar e incentivar el trabajo en equipo, la integración y la comunicación en pro a la organización, inspirados en la inteligencia emocional para canalizar las emociones.

2. Crear planes de capacitación para todos los empleados de la empresa de modo que todos sepan las funciones de todos para facilitar así la delegación de las funciones necesarias para incentivar el cambio y adecuar el clima organizacional.

3. Velar por la participación de los empleados y la integración total de los colaboradores de la organización, así como también, de los gerentes para una mejor comunicación e interacción laboral.

4. Revisar y hacer seguimiento para los trámites de jubilación de personas que cumplen y exceden el número de años de servicios en la organización.

\section{Referencias}

Arias, F. (2012). El proyecto de investigación: Introducción a la metodología científica. 6ta Edición, Colección General, ISBN: 980-078529-9. Caracas, Venezuela: Editorial Episteme.

Gil, H. (2007). El enfoque etnometodológico en la investigación científica. Liberabit, 13(13), 89-91, e-ISSN: 1729-4827, Versión impresa. Recuperado de:

http://www.scielo.org.pe/scielo.php?script=sci arttext\&pid=S1729$\underline{48272007000100011}$

Hurtado, I., \& Toro, J. (2010). Paradigmas y Métodos de Investigación en tiempos de cambio. 5ta. Edición. ISBN: 980-328-413-4. Valencia, Carabobo, Venezuela: Episteme Consultores Asociados, C.A.

Ivancevich, J., Konopaske, R., \& Matteson, M. (2006). Comportamiento

Organizacional. Séptima Edición, ISBN: 970-10-5623-X. México, D.F.: 
McGraw-Hill/Interamericana Editores, S.A. de C.V. Recuperado de: https://www.upbvirtual.net/upbvirtual/pluginfile.php/164015/course/over viewfiles/Comportamiento\%20organizacional\%207ed\%20lvancevich.p df? forcedownload $=1$

Gil, P. (2005a,b). El síndrome de quemarse por el trabajo (burnout). Una enfermedad laboral en la sociedad del bienestar. 1ra Edición, Colección de Psicología, ISBN: 978-84-368-1949-6. Madrid, España: Ediciones Pirámide, 192 págs.

Miravalles, J. (2009). Cuestionario de Maslach Burnout Inventory. San Juan de la Cruz, Zaragoza: Gabinete Psicológico. Recuperado de: http://www.javiermiravalles.es/sindrome\%20burnout/Cuestionario\%20d e\%20Maslach\%20Burnout\%20Inventory.pdf

Schwartzmann, L. (2004). Estrés laboral, síndrome de desgaste (quemado), depresión: ¿Estamos hablando de lo mismo? Ciencia \& trabajo, 6(14), 174-184, e-ISSN: 0718-0306. Recuperado de: http://bases.bireme.br/cgi-

bin/wxislind.exe/iah/online/?IsisScript=iah/iah.xis\&base=LILACS\&lang= p\&nextAction=Ink\&exprSearch=420804\&indexSearch=ID

Stora, J. (1991). El Estrés, ¿Qué sé? Primera Edición Francesa, ISBN 13: 9789682001796. Mixcoac, México, D.F.: Publicaciones Cruz O., S.A.

Suárez, D., \& Contreras, Y. (2010). Síndrome de Burnout: comparación entre alumnos y profesor-odontólogo en la Facultad de Odontología de la Universidad de Los Andes. Acta Bioclínica, 3(5), 158-177, e-ISSN: 2244-8136. Recuperado de:

http://erevistas.saber.ula.ve/index.php/actabioclinica/article/view/4409 


\section{Carlos Liborio Camacho Quintero}

e-mail: ccamacho@ula.ve; clcamachoq71@gmail.com

Nacido en Mérida, estado Mérida, Venezuela, el 25 de marzo del año 1971. Post-Doctor en Gerencia para el Desarrollo Humano (ULA); Doctor en Gerencia Avanzada; Doctorando en Ciencias de la Educación; Magíster Scientiarum en Gerencia Empresarial; Especialista en Telemática e Informática en Educación a distancia (EaD); Ingeniero de Sistemas; Licenciado en Administración de Empresas; Técnico Superior Universitario en Informática; Técnico Superior Universitario en Administración Mención Mercadotecnia. Componente Docente de la Universidad de los Andes (ULA); Diplomado en Componente Docente en Educación a Distancia; Diplomado Internacional en TIC; Investigador PEII ONCTI - PEII ULA; Experto de la Fundación para la Actualización Tecnológica de Latinoamérica (FATLA) en: Educación Virtual, Administración Web y Comercio Electrónico; dominio de inglés técnico; manejo de programas bajo ambiente Windows; instructor Linux y Web Master.

El contenido de este manuscrito se difunde bajo una Licencia de Creative Commons ReconocimientoNoComercial-Compartirlgual 4.0 Internacional 